\title{
Efeitos da ibopamina 2\% tópica nos resultados da campimetria visual computadorizada
}

\author{
Effects of 2\% ibopamine eye drops on computerized visual field results
}

\author{
Mara Agi Pigini ${ }^{1}$ \\ Marcos Pereira Ávila² \\ Leopoldo Magacho ${ }^{3}$
}

\begin{tabular}{|c|}
\hline RESUMO \\
\hline 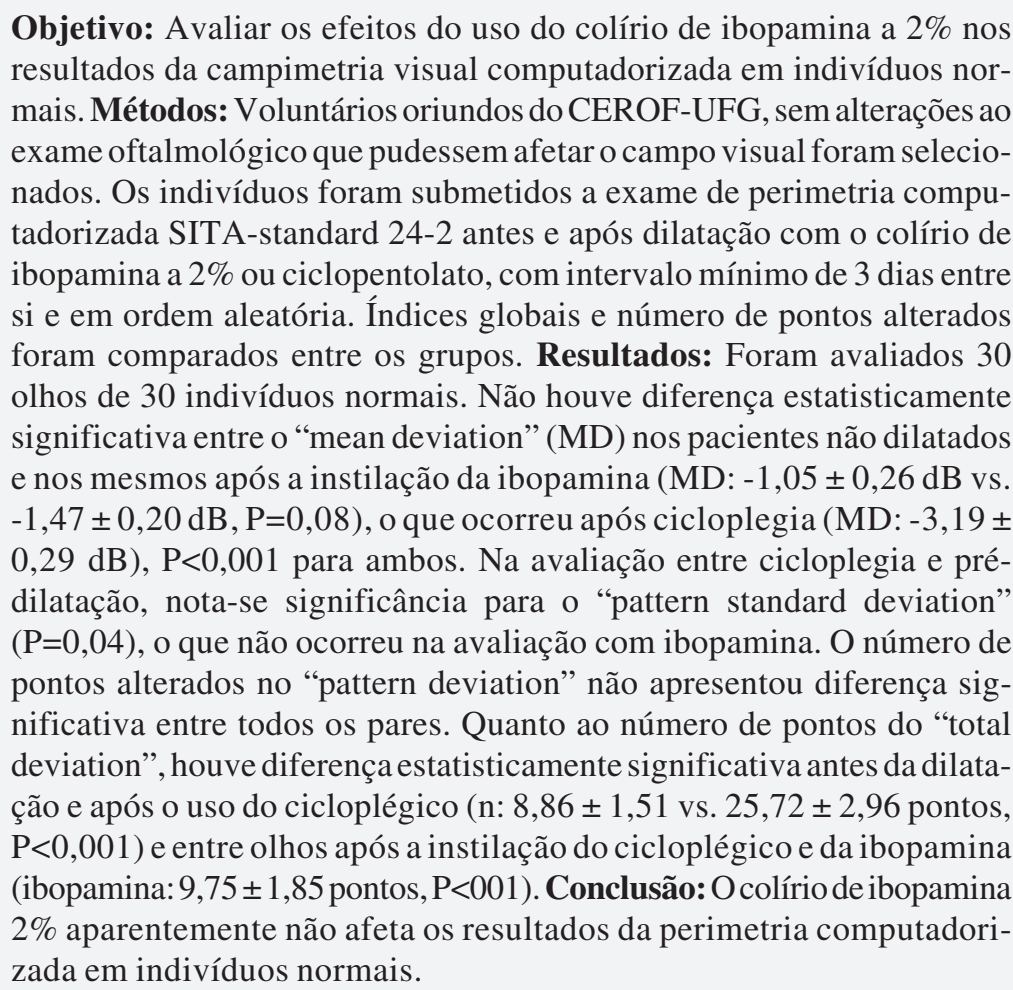 \\
\hline
\end{tabular}

Descritores: Ciclopentolato/administração \& dosagem; Glaucoma/diagnóstico; Hipertensão ocular; Pressão intraocular/efeitos de drogas; Perimetria Referência em Oftalmologia - CEROF da Universidade Federal de Goiás - UFG - Goiânia (GO) - Brasil.

Pós-graduanda em Ciências da Saúde (Oftalmologia) da Universidade Federal de Goiás - UFG - Goiânia (GO) Brasil.

${ }^{2}$ Doutor, Professor e Chefe do Centro de Referência em Oftalmologia - CEROF da UFG - Goiânia (GO) - Brasil. ${ }^{3}$ Doutor, Professor de Pós-graduação; Coordenador do Setor de Glaucoma e Coordenador Acadêmico do CEROF da UFG - Goiânia (GO) - Brasil.

Endereço para correspondência: Mara Agi Pigini Rua Hugo de Carvalho Ramos, 305 - Anápolis (GO) CEP 75110-320

E-mail: maraagi@hotmail.com

Os autores declaram não possuir qualquer interesse comercial nos produtos citados no texto.

Recebido para publicação em 24.06.2008

Última versão recebida em 22.02.2009

Aprovação em 16.03.2009

\section{INTRODUÇÃO}

INTRODUÇÃO

Estima-se que o glaucoma afetará mais de 60 milhões de pessoas no mundo até 2010, chegando a quase 80 milhões em 2020, representando a principal causa de cegueira irreversível ${ }^{(1)}$. Essa característica torna de grande importância o exame oftalmológico de rotina, para o acompanhamento e detecção precoce da doença, que se manifesta de forma variada entre os indivíduos, apresentando diversos subtipos ${ }^{(2)}$.

Dentro dessa investigação, um exame extremamente valioso é a perimetria visual computadorizada. Entretanto, esse exame não pode ser realizado quando há dilatação pupilar por ter a sua sensibilidade alterada. Isso obriga o paciente a um novo retorno, já que a avaliação estereoscópica sob midríase do disco óptico é necessária na primeira visita. O uso de um colírio midriático não-cicloplégico, que não afete os resultados da perimetria seria
\end{abstract}


o ideal. Se, ainda, for útil como teste provocativo, completaria a propedêutica ideal. Dentro dessa expectativa, o colírio de ibopamina surge como uma possibilidade de responder a essas exigências.

A ibopamina é uma pró-droga da N-metildopamina, hidrolisada em epinina, com atividade nos receptores adrenérgicos e dopaminérgicos ${ }^{(3)}$. Apresenta uma ação midriática não-cicloplégica $^{(4)}$ devido a suas propriedades alfa-adrenérgicas ${ }^{(5)}$, e é capaz de induzir a um aumento transitório da pressão intraocular (PIO) por aumentar a produção de humor aquoso através de sua ação nos receptores D1 dopaminérgicos ${ }^{(6-8)}$.

Esse aumento significativo da PIO ocorre, na maioria dos casos, apenas em olhos glaucomatosos, que geralmente apresentam alteração nas vias de escoamento do humor aquoso ${ }^{(9)}$. O mesmo teste em olhos normais não foi capaz de elevar a PIO a níveis estatisticamente significativos ${ }^{(3,5)}$, levando a uma boa relação sensibilidade/especificidade na diferenciação entre olhos normais e glaucomatosos ${ }^{(10-14)}$.

O efeito midriático da ibopamina $2 \%$ supera, em termos de medida da dilatação pupilar, aquele produzido pela tropicamida $1 \%$ e pela fenilefrina $10 \%{ }^{(15)}$. Ao contrário da tropicamida e da fenilefrina, que produzem uma redução significativa da sensibilidade à realização da perimetria visual computadorizada $^{(16-18)}$, a ibopamina não altera a refração nem a acuidade visual do paciente ${ }^{(8,19)}$.

Dessa forma, espera-se que a ibopamina não afete os resultados da perimetria computadorizada, por não provocar cicloplegia, ou ainda, que essa alteração seja mínima, induzida exclusivamente pela importante midríase gerada pela droga. $\mathrm{O}$ presente estudo visa verificar se o colírio de ibopamina, usado da maneira como preconizado no seu teste provocativo ${ }^{(6,12)}$, altera os resultados da campimetria computadorizada em indivíduos normais.

\section{MÉTODOS}

O presente estudo, após aprovação pelo comitê de ética em pesquisa médica do Hospital das Clínicas da Universidade Federal de Goiás (UFG), e consentimento livre e informado por parte dos participantes, selecionou indivíduos normais provenientes do Centro de Referência em Oftalmologia (CEROF) da UFG, constituídos de médicos, funcionários e voluntários, que tiveram apenas um dos olhos selecionados para a análise. Nos casos em que ambos os olhos eram igualmente elegíveis, foi incluído apenas o olho direito.

Esses voluntários foram submetidos a uma avaliação oftalmológica completa, incluindo a medida da acuidade visual sem e com correção, biomicroscopia, tonometria de aplanação com tonômetro de Goldmann e biomicroscopia de fundo de olho usando uma lente de 78 dioptrias.

Para a inclusão, a acuidade visual sem correção deveria ser melhor que 20/30, limitando as possíveis ametropias. Nos casos em que houve a necessidade de correção de algum erro refracional, essa mesma correção foi obrigatoriamente realiza- da em todas as etapas do estudo. A pressão intraocular deveria ser menor que $21 \mathrm{mmHg}$ em duas medidas diferentes, e a biomicroscopia não poderia apresentar quaisquer alterações que pudessem interferir na tonometria ou no resultado da perimetria visual computadorizada, como, por exemplo, catarata, leucomas e ceratocone. À fundoscopia, exigiu-se ausência de alterações glaucomatosas do disco óptico.

Foram excluídos deste protocolo os indivíduos que apresentaram acuidade visual pior que 20/30 sem correção, míopes ou hipermétropes. Também se excluiu aqueles que apresentaram pressão intraocular maior ou igual a $21 \mathrm{mmHg}$ em pelo menos uma das medidas, ou ainda qualquer anormalidade à avaliação da biomicroscopia, cirurgia ocular prévia, doenças retinianas, doenças neurológicas que pudessem interferir nos resultados do campo visual computadorizado, e outras doenças sistêmicas incapacitantes (insuficiência cardíaca, doença vascular cerebral ou demência).

Os indivíduos selecionados realizaram uma perimetria computadorizada SITA-fast 24-2 no olho não incluso para minimizar o efeito aprendizado ${ }^{(20)}$. Definiu-se realizar primeiro a campimetria sem dilatação pupilar em ambos os grupos a fim de se reduzir o número de visitas por parte dos voluntários, tornando necessárias apenas duas sessões para completar o protocolo do estudo. A ordem de realização das demais campimetrias foi aleatória, definida por meio de um sorteio prévio. Os participantes foram divididos aleatoriamente, também por meio de um sorteio, em dois grupos, a depender da ordem dos colírios usados para a dilatação pupilar.

Os integrantes do primeiro grupo (Grupo 1) realizaram perimetria computadorizada SITA-standard 24-2 no olho do estudo, seguida da dilatação pupilar com o colírio de ibopamina $2 \%$ ( 2 gotas com intervalo de 5 minutos) e, após 1 hora, repetiram o mesmo exame de perimetria no olho selecionado. Com o intervalo mínimo de 3 dias, foram novamente submetidos a dilatação pupilar, desta vez com colírio cicloplégico (ciclopentolato - 1 gota de 5 em 5 minutos, 3 vezes), realizando um novo campo SITA-standard após 1 hora.

Os indivíduos do segundo grupo (Grupo 2) realizaram, igualmente, uma perimetria computadorizada SITA-standard 24-2 no olho do estudo, seguida da dilatação pupilar inicialmente com o colírio de ciclopentolato ( 3 gotas com intervalo de 5 minutos), e, após 1 hora, repetiram o mesmo exame de perimetria no olho selecionado. Com o intervalo mínimo de 3 dias, foram novamente submetidos a dilatação pupilar, desta vez usando a ibopamina.

A análise estatística foi realizada através do software SPSS, versão 11.5 (SPSS Inc, Chicago, IL, EUA). O teste de Kolmogorov-Smirnov foi utilizado para se testar a normalidade das amostras. O teste t de Student pareado foi utilizado para a comparação aos pares dos índices globais "mean deviation" (MD) e "pattern standard deviation" (PSD) do campo visual, bem como do número de pontos alterados dos gráficos do "total deviation" (TD) e "pattern deviation" (PD). No presente estudo, valores de $\mathrm{p}<0,05$ foram considerados como significativos. 


\section{RESULTADOS}

Foram incluídos 30 participantes, sendo 15 no grupo 1 e 15 no grupo 2. A idade média dos participantes foi de 24,63 anos (DP: $\pm 6,58$ anos), sendo 11 do sexo masculino e 19 do sexo feminino.

Não houve diferença estatisticamente significativa entre o MD nos indivíduos não dilatados e nos mesmos após a instilação da ibopamina (MD: $-1,05 \pm 0,26 \mathrm{~dB}$ vs. $-1,47 \pm 0,20 \mathrm{~dB}$, $\mathrm{P}=0,08)$. Entretanto, após cicloplegia (MD: $-3,19 \pm 0,29 \mathrm{~dB}$ ), nota-se diferença, tanto em relação ao uso da ibopamina, quanto ao exame sem o uso de colírios $(\mathrm{P}<0,001$ para ambos), conforme apresentado na figura 1.

Em relação ao PSD, não houve diferença quando comparados os indivíduos antes da dilatação pupilar $(1,69 \pm 0,09 \mathrm{~dB}) \mathrm{e}$ após o uso de ibopamina $(1,61 \pm 0,09 \mathrm{~dB}, \mathrm{P}=0,4)$, e comparando-se o cicloplégico $(1,45 \pm 0,05 \mathrm{~dB})$ com a ibopamina $(\mathrm{P}=0,09)$, como mostra na figura 2. Entretanto, houve diferença estatisticamente significativa ao se comparar os valores pré-dilatação com os resultados após o uso de cicloplégico $(\mathrm{P}=0,04)$.

Quanto ao número de pontos do TD, houve diferença estatisticamente significativa entre os seguintes pares avaliados: $8,86 \pm 1,51$ pontos (sem dilatação) vs. $25,72 \pm 2,96$ pontos (ciclo, $\mathrm{P}<0,001)$, e ciclo vs. ibopamina $(9,75 \pm 1,85$ pontos, $\mathrm{P}<0,001)$. Entretanto, não houve significância ao se comparar o exame com o uso da ibopamina vs. exame sem dilatação $(\mathrm{P}=0,6)$.

$\mathrm{Na}$ avaliação dos pontos do $\mathrm{P}$, não houve diferença estatisticamente significativa ao se comparar o exame pré-dilatação $(5,13 \pm 0,80$ pontos $)$ com o uso do cicloplégico $(4,34 \pm 0,75$ pontos, $\mathrm{P}=0,4)$. $\mathrm{O}$ mesmo ocorreu na comparação do exame pré-dilatação com o exame após o uso da ibopamina $(4,77 \pm$ 0,76 pontos, $\mathrm{P}=0,8$ ) bem como na comparação entre os resultados obtidos com o uso da ibopamina versus os obtidos com o cicloplégico $(\mathrm{P}=0,7)$.

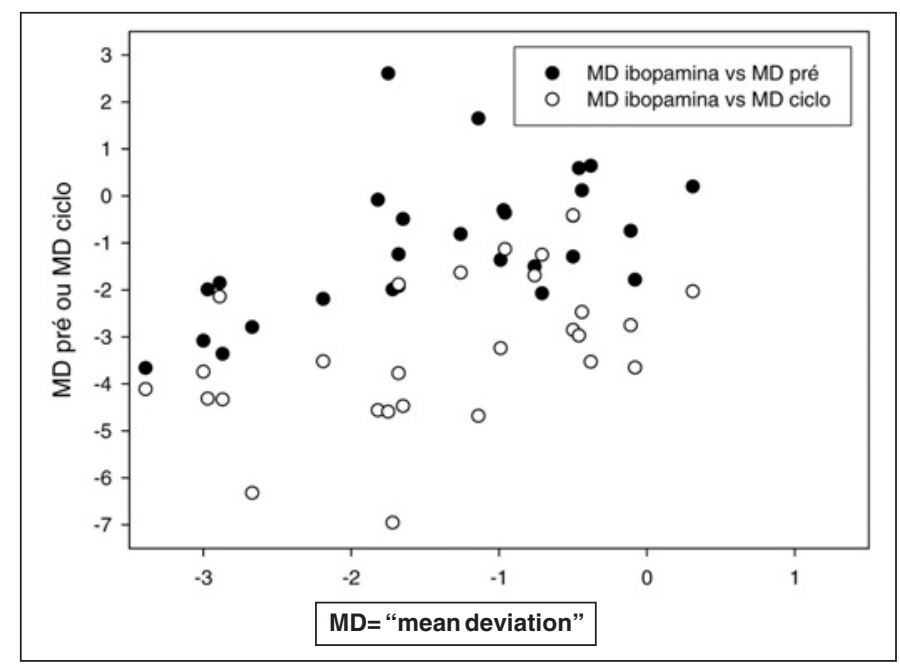

Figura 1 - Correlação entre o MD pré-dilatação, MD pós-cicloplegia e MD pós-ibopamina

\section{DISCUSS ÃO}

O objetivo desse trabalho foi o de avaliar se o uso do colírio de ibopamina $2 \%$, indicado como teste provocativo para o glaucoma, altera os resultados da campimetria computadorizada.

Ao se avaliar o número de pontos alterados no gráfico do TD, pode-se perceber diferença significativa apenas com o uso do cicloplégico. A ibopamina não foi capaz de provocar alterações significativas neste gráfico. O TD compara os resultados obtidos no exame do participante com indivíduos normais na mesma faixa etária, e reflete perdas da sensibilidade geral, indicando diferenças ponto a ponto nas regiões avaliadas ${ }^{(21)}$.

O número de pontos estatisticamente alterados no gráfico do "pattern deviation" (PD), que é calculado corrigindo-se as influências da redução global da sensibilidade (como catarata, miose ou cicloplegia) ${ }^{(22-23)}$, não apresentou diferença significativa entre os grupos. Esses resultados eram esperados já que elimina a influência da dilatação pupilar e cicloplegia provocadas pelo colírio cicloplégico.

$\mathrm{Na}$ avaliação dos efeitos da ibopamina, pode-se notar que não houve alteração da sensibilidade global nos testes realizados, já que os valores do "mean deviation" (MD) não tiveram uma variação estatisticamente significativa (MD: $-1,05 \pm$ $0,26 \mathrm{~dB}$ vs. $-1,47 \pm 0,20 \mathrm{~dB}, \mathrm{P}=0,08$ ). No gráfico do $\mathrm{MD}$ (Figura $1)$, percebe-se que os valores pré-dilatação e com ibopamina são muito semelhantes, já que a nuvem de dispersão tem formato mais parecido com uma reta. Entretanto, ao se realizar o teste com o cicloplégico, nota-se uma diminuição significativa nos valores do MD, refletindo alteração na sensibilidade global dos particiantes provocada pela cicloplegia, e já previamente relatada ${ }^{(17-18)}$. Essa alteração pode ser notada como uma nuvem de dispersão com um formato mais espalhado. Esses resultados são esperados, já que o MD deriva da média dos pontos do $\mathrm{TD}^{(24-25)}$.

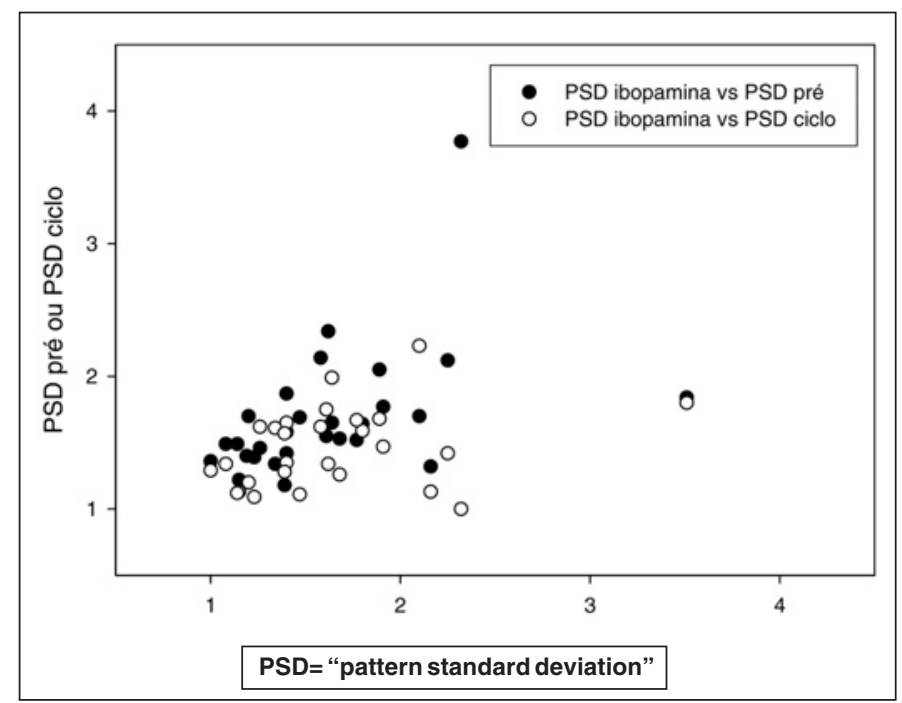

Figura 2 - Correlação entre o PSD pré-dilatação, PSD pós-cicloplegia e PSD pós-ibopamina 
Ao se comparar o PSD, a diferença entre os pares avaliados não foi notada, já que sua fórmula inclui mecanismos para filtrar as baixas globais de sensibilidade ${ }^{(22,24-25)}$, o que aparece graficamente como nuvens de dispersão semelhantes (Figura 2). Além disso, o PSD reflete a média ponderada do gráfico do "pattern deviation" (PD), e sugere perdas localizadas, características do glaucoma, o que não ocorre com a cicloplegia.

Esses resultados ajudam a corroborar o fato da ibopamina ser um potente midriático e sugerem propriedades não cicloplégicas da ibopamina. Entretanto, nenhuma medida óptica ou de acomodação foi realizada para confirmação.

Os resultados são reforçados pelo fato de ter sido realizada uma perimetria visual computadorizada prévia no olho contralateral, visando reduzir o efeito aprendizado ${ }^{(20)}$, que poderia interferir nos testes. Nesse mesmo sentido, os voluntários selecionados foram divididos em dois grupos, alterando a ordem dos exames, já que teoricamente os exames realizados posteriormente contariam com indivíduos mais treinados. Além disso, tentou-se reduzir ao mínimo o viés de inclusão na realização do exame de campo visual, que poderia ocorrer se outras alterações oftalmológicas estivessem presentes, incluindo-se apenas indivíduos normais. Indivíduos com diagnóstico de glaucoma, por sua vez, poderiam apresentar alterações perimétricas próprias da doença, como flutuação a curto prazo ${ }^{(24)}$, alterando os resultados produzidos exclusivamente pela ibopamina.

A inclusão de um segundo grupo controle (ciclopentolato), visa mostrar que indivíduos normais, submetidos à dilatação com cicloplegia, têm o seu campo visual alterado. Na ibopamina, apesar da midríase acentuada, não houve mudança nos resultados gerais, reforçando a sugestão de ausência de propriedade cicloplégica para essa droga.

Uma avaliação oftalmológica da acuidade visual, tonometria, biomicroscopia, seguidas de gonioscopia e dilatação pupilar com ibopamina em casos suspeitos de glaucoma, permite já selecionar aqueles com um risco em potencial. A mesma ibopamina, simultaneamente, produz uma midríase que permite a avaliação fundoscópica com estereopsia, com atenção especial ao disco óptico e camada de fibras nervosas da retina.

Os resultados apresentados sugerem que o colírio de ibopamina a $2 \%$ não influencia a realização da perimetria computadorizada, Dessa maneira, mesmo com a importante midríase obtida com a instilação da mesma, a ausência de cicloplegia aparentemente poderia permitir que o exame campimétrico seja realizado sem interferência, seguindo-se uma rotina propedêutica sem a necessidade de visitas adicionais, otimizando o tempo e eventuais custos para o indivíduo e o serviço de saúde. Como se trata de estudo pioneiro, sugere-se a realização de avaliações com um número maior de participantes, incluindo na amostra pacientes glaucomatosos.

\section{CONCLUSÃO}

O colírio de ibopamina a $2 \%$ não alterou os resultados da perimetria computadorizada em indivíduos normais.

\section{ABSTRACT}

Purpose: To evaluate the influence of $2 \%$ ibopamine eye drops on the results of computerized visual field exams. Methods: Normal volunteers from CEROF-UFG were selected, with no variance in the ophthalmologic examination that could affect the visual field test. The volunteers underwent computerized visual field test before and after dilation with $2 \%$ ibopamine eye drop or cyclopentolate, with a minimum interval of three days between them and in a random order. Global indices and number of altered points were compared between the groups. Results: Thirty eyes of 30 normal individuals were selected. There was no statistically significant difference on Mean Deviation (MD) before and after dilation with ibopamine (MD: $-1.05 \pm 0.26 \mathrm{~dB}$ vs. $-1.47 \pm 0.20 \mathrm{~dB}, \mathrm{P}=0.08$ ). However, after cycloplegia (MD: $-3.19 \pm 0.29 \mathrm{~dB}$ ), there was a significant difference on $\mathrm{MD}(\mathrm{P}<0.001$ for both ibopamine and pre-dilation). No significant difference was detected in the Pattern Standard Deviation when comparing ibopamine with pre-dilation and cycloplegia values, but it was statistically significant comparing pre-dilation to cycloplegia $(\mathrm{P}=0.04)$. The number of altered points in the Pattern Deviation graphic were not significant comparing all pairs. There was a statistically significant difference in the number of altered points in the total deviation graphic before dilation and after cycloplegia (n: $8.86 \pm 1.51$ vs. $25.72 \pm 2.96$ points, $\mathrm{P}<0.001$ ), and comparing cycloplegia with ibopamine (ibopamine: $9.75 \pm 1.85$ points, $\mathrm{P}<0.001$ ). Conclusion: Ibopamine $2 \%$ eye drops seem to not modify the results of visual field tests in normal individuals.

Keywords: Cyclopentolate/administration \& dosage; Glauco$\mathrm{ma} /$ diagnosis; Ocular hypertension; Intraocular pressure/drug effects; Perimetry

\section{REFERÊNCIAS}

1. Quigley HA, Broman AT. The number of people with glaucoma worldwide in 2010 and 2020. Br J Ophthalmol. 2006;90(3):262-7. Comment in: Br J Ophthalmol. 2006;90(3):253-4.

2. Shields B, Allingham R, Damji KF, Freedman S, Moroi SE, Shafranov G. Shields' textbook of glaucoma. $5^{\text {th }}$ ed. Philadelphia: Lippincott Williams \& Wilkins; 2005. p.155-62.

3. Azevedo H, Ciarniello MG, Rosignoli MT, Dionisio P, Cunha-Vaz J. Effects of ibopamine eye drops on intraocular pressure and aqueous humor flow in healthy volunteers and patients with open-angle glaucoma. Eur J Ophthalmol. 2003; 13(4):370-6.

4. Marchini G, Babighian S, Tosi R, Perfetti S, Bonomi L. Effects of $2 \%$ ibopamine on pupil, refraction, anterior segment anatomy and intraocular pressure. J Ocul Pharmacol Ther. 2001;17(3):215-23.

5. Giuffrè I, Taverniti L, Di Staso S. The effects of $2 \%$ ibopamine eye drops on the intraocular pressure and pupil motility of patients with open-angle glaucoma. Eur J Ophthalmol. 2004;14(6):508-13.

6. De Gregorio F, Pecori Giraldi J, Pannarale L, Saccucci S, Virno M. Ibopamine in glaucoma diagnostics: a new pharmacological provocative test. Int Ophthalmol. 1996-1997;20(1-3):151-5.

7. Virno M, Taverniti L, De Gregorio F, Sedran L, Longo F. Increase in aqueous humor production following D1 receptors activation by means of ibopamine. Int Ophthalmol. 1996-1997;20(1-3):141-6.

8. Gelmi C, Palazzuolo A, Lucchetti M, Trimarchi F. Pupillographic evaluation 
of the mydriatic effect of ibopamine solution. Int J Clin Pharmacol Ther Toxicol. 1989;27(7):346-51.

9. Hamard P, Valtot F, Sourdille P, Bourles-Dagonet F, Baudouin C. Confocal microscopic examination of trabecular meshwork removed during ab externo trabeculectomy. Br J Ophthalmol. 2002;86(9):1046-52.

10. Magacho L, Lima FE, Costa ML, Fayad FA, Guimarães NL, Avila MP. Ibopamine provocative test and glaucoma: consideration of factors that may influence the examination. Curr Eye Res. 2004;28(3):189-93.

11. De Gregorio F, Pecori-Giraldi J, De Stefano C, Virno M. Correlation between ocular hypertension induced by ibopamine and perimetric defect in primary open-angle glaucoma. Eur J Ophthalmol. 1997;7(2):152-5.

12. Lima FE, Guimarães NLD, Santos LM, Costa LP, Ávila M. Ibopamina tópica na propedêutica do glaucoma. Rev Bras Oftalmol. 2002;61(2):109-13.

13. Magacho L, Costa ML, Dessimoni A, Avila MP. Comparison between the 1 percent and 2 percent ibopamine provocative test in primary open-angle glaucoma patients: sensitivity, specificity and tolerability. Arq Bras Oftalmol. 2006; 69(5):695-9.

14. Magacho L, Costa ML, Lima FE, Magacho B, Ávila MP. Análogos das prostaglandinas diminuem a sensibilidade do teste provocativo da ibopamina no glaucoma. Arq Bras Oftalmol. 2006;69(2):193-6.

15. Marchini G, Babighian S, Tosi R, Perfetti S, Bonomi L. Comparative study of the effects of $2 \%$ ibopamine, $10 \%$ phenylephrine, and $1 \%$ tropicamide on the anterior segment. Invest Ophthalmol Vis Sci. 2003;44(1): 281-9.
16. Park HJ, Youn DH. Quantitative analysis of changes of automated perimetric thresholds after pupillary dilation and induced myopia in normal subjects. Korean J Ophthalmol. 1994;8(2):53-60.

17. Lindenmuth KA, Skuta GL, Rabbani R, Musch DC, Bergstrom TJ. Effects of pupillary dilation on automated perimetry in normal patients. Ophthalmology. 1990;97(3):367-70.

18. Mendivil A. Influence of a dilated pupil on the visual field in glaucoma. J Glaucoma. 1997;6(4):217-20.

19. Virno M, Taverniti L, Pecori-Giraldi J. Ibopamina: nuevo midriatico non cicloplegico (nota preliminare). Boll Ocul. 1986;65:1135-6.

20. Heijl A, Lindgren G, Olsson J. The effect of perimetric experience in normal subjects. Arch Ophthalmol. 1989;107(1):81-6.

21. Heijl A, Patella VM. Essential perimetry. $3^{\text {rd }}$ ed. Dublin: Carl Zeiss Meditec; 2002. p.45-7.

22. Shields B, Allingham R, Damji KF, Freedman S, Moroi SE, Shafranov G. Shields' textbook of glaucoma. $5^{\text {th }}$ ed. Philadelphia: Lippincott Williams \& Wilkins; 2005. p.124-5.

23. Heijl A, Patella VM. Essential perimetry. $3^{\text {rd }}$ ed. Dublin: Carl Zeiss Meditec; 2002. p. 10 .

24. Shields B, Allingham R, Damji KF, Freedman S, Moroi SE, Shafranov G. Shields' textbook of glaucoma. $5^{\text {th }}$ ed. Philadelphia: Lippincott Williams \& Wilkins; 2005. p.129-30.

25. Heijl A, Patella VM. Essential perimetry. $3^{\text {rd }}$ ed. Dublin: Carl Zeiss Meditec; 2002. p.55.

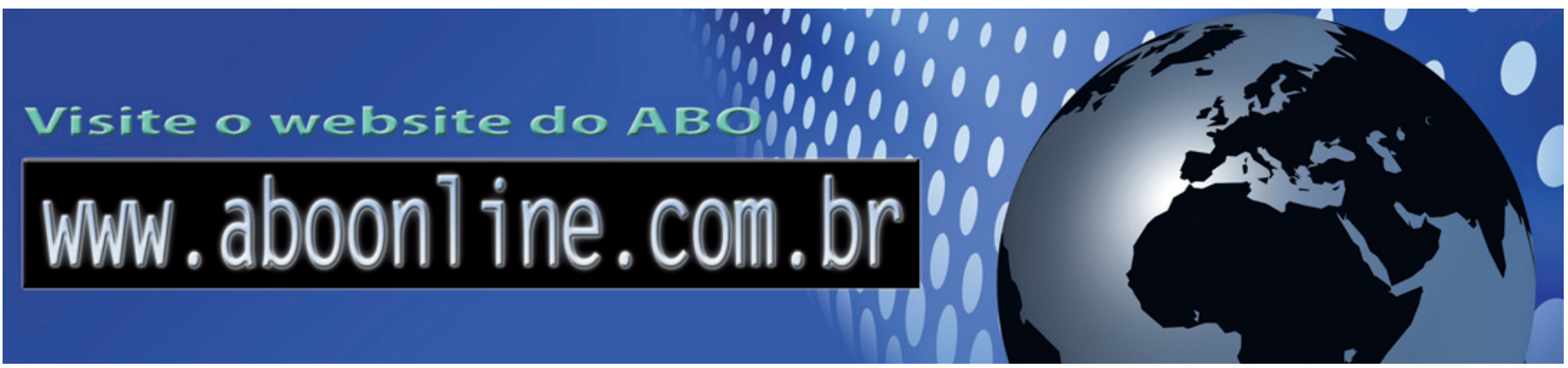

